\title{
Methods of Measuring Influence of Bank Customer Using Social Network Model
}

\author{
Huaqing Mao, Xinyuan Jin*, Li Zhu \\ Oujiang College, Wenzhou University, Wenzhou, China

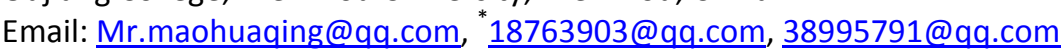

Received 9 March 2015; accepted 5 April 2015; published 8 April 2015

Copyright $@ 2015$ by authors and Scientific Research Publishing Inc.

This work is licensed under the Creative Commons Attribution International License (CC BY). http://creativecommons.org/licenses/by/4.0/

(c) (i) Open Access

\begin{abstract}
As the development of economic today, Bank business deeply integrates into our lives. Thousands of transactions generate the large amounts of data. Traditional bank customer management system simply classifies and counts these data, and the system is deficient in customer relationship management. However, the social network model can provide the influence between the bank customers for the bank. In this paper, we construct a social network by calculating the relationship between bank customers. In order to explore how bank customers in the bank customer network affect each other, we use three indicators: the average path length $L$, clustering coefficient $C$ and degree distribution $p(x)$ to conduct a comprehensive analysis. Consequently, we find that there evidently exist influential customers in this network.
\end{abstract}

\section{Keywords}

Social Network Model, Influence Analysis, Bank Customer Management

\section{Introduction}

With the progress of financial science and technology, a large number of efficient computer application systems arise at the historic moment. Those applications are used in bank business widely, which greatly improves the service quality, and benefits for the efficiency and quality of service processing [1]-[5]. In the meantime, the rapid developments of information technology and cyber economy cause the strong business competition of bank. The scale advantage formed by expansion of financial organization is fading away. The development of bank is turning from pursuing scale advantage into digging customer advantage, and provides the different service and production to different customers [6]-[9]. Therefore, how to determine the influence and importance of customer and identify the value of customer become the core issues. The effective method and the theory to dis-

${ }^{*}$ Corresponding author.

How to cite this paper: Mao, H.Q., Jin, X.Y. and Zhu, L. (2015) Methods of Measuring Influence of Bank Customer Using Social Network Model. American Journal of Industrial and Business Management, 5, 155-160.

http://dx.doi.org/10.4236/ajibm.2015.54017 
tinguish the customer have become the key factor that bank generates profit and promotes the nuclear competitive power. As the increasing sophistication of the research method, social network analysis has caused wide public concern in many research fields over the recent years. It proffers a new research method for bank customer management [10]-[12].

\section{Assumptions and Conventions}

\subsection{Assumptions}

In order to ensure the effective operation of our model, we made the following assumptions:

- The customer does not have any financial misconduct.

- The influence of each customer in the social network is equivalent.

- Each customer made the same influence to any of the other customers.

- The relationship between the influence of the customer and the frequency of his cooperation with Bank is linear.

\subsection{Conventions}

In our social network model, we made the following conventions:

- The average path length $\mathrm{L}$ is the mean value of he path length in the network between any pair of nodes.

- Clustering coefficient $C_{i}$. It describes the network connection nodes in the network between neighboring nodes connected to measure the likelihood of any one of the network nodes. In other words, it is the ratio that neighboring nodes are actually connected to edges and the theoretical side edges.

- Degree of node degree distribution $p(k)$ as the probability of node $k$. That is the number of nodes of degree $k$ appears with the number of nodes in all degrees appeared ratio.

- Degree centrality $C_{D}$. By calculating the point of the degree, makes clear who is the central character in the team, that is, who is the most powerful and influential person in the team. We use the number of contact to indicate the degree of the nodes, for node $=a_{i}$, the degree of the nodes $=d\left(a_{i}\right)$.

- Degree centrality with the weights $C_{w}\left(a_{i}\right)$. Sowis made to serve as the weights of the node $a_{i}$ considering the influence by the weights of the direct link node of $a_{i}$ upon $a_{i}$.

\section{Social Network Analysis of Bank Customer}

\subsection{Establish the Model}

Studying social network of bank customer, namely the establishment of the social network model of the relationship (hereinafter referred to as social network model). In order to establish the social network model, we need to get all inter-linked customer with a known customer named Li. Those customers who are not directly connected with Li should be excluded from the model. In addition, because of their influence can be ignored, customers who are not connected with most of the customers (secondary customers of connected components) should also be excluded from the model. The above approach, simplifying the model won't have much impact on the results. Finally, the remaining customers are sorted in alphabetical order numbers. Steps are as follows:

Step 1: Importing data.

Step 2: Delete customers who are not directly connected with $\mathrm{Li}$.

Step 3: Get the link between the customers (not including Li).

Step 4: Keep the main branch connectivity (Delete the secondary branch connectivity).

Step 5: The customers are sorted in alphabetical number.

Step 6: Draw network diagrams (Shown in Figure 1).

The data of bank records have two types (Shown in Table 1 and Table 2), one is the transfer records, and the other is loan records. Those two types of records contain the information about the relationship between the bank customers.

To build a social network for analysis, we select the characteristic attributes of several networks as following sections:

\subsection{Average Path Length}

Two nodes of the path length between $i$ and $j$ in the network $l_{i j}$ is defined as the number of edges connected to 


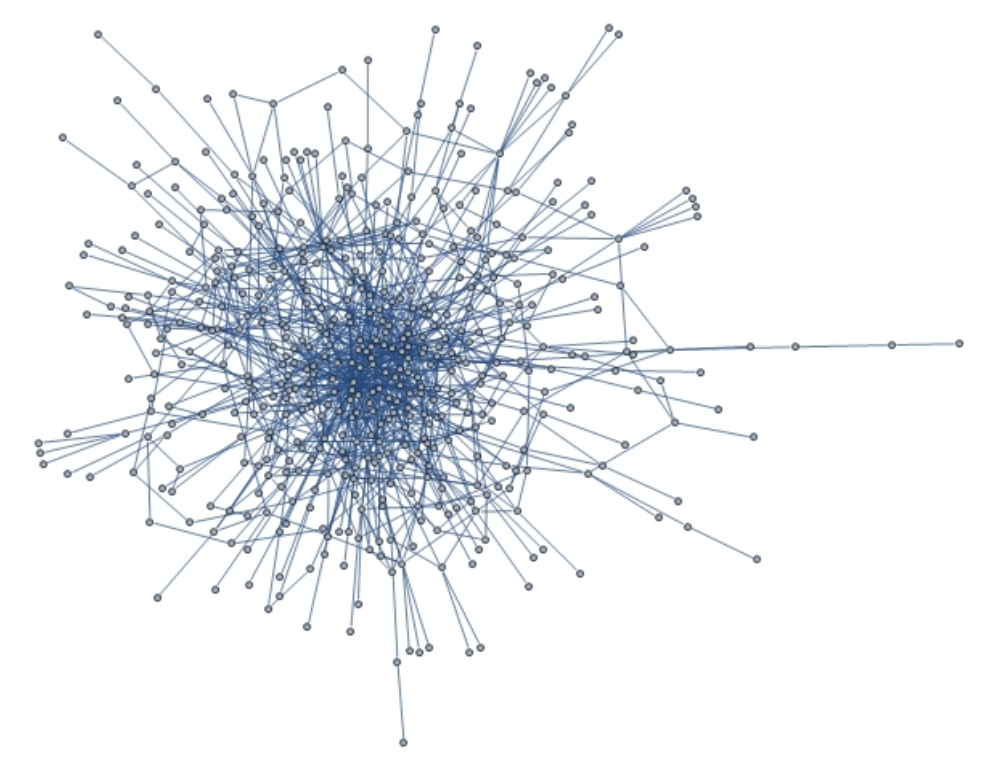

Figure 1. Network diagram.

Table 1. Transfer records.

\begin{tabular}{cccc}
\hline Bank & Transfer Account & Receive Account & Transfer Amount (RMB) \\
\hline Bank of China & $\mathrm{Li}$ & Wang & 3000 \\
Bank of China & $\mathrm{Li}$ & Zhang & 3500 \\
$\ldots$ & $\ldots$ & $\ldots$ & $\ldots$ \\
\hline
\end{tabular}

Table 2. Loan records.

\begin{tabular}{ccc}
\hline Bank & Loan Group & Transfer Amount (RMB) \\
\hline Bank of China & $\mathrm{Li}, \mathrm{Xu}$ & 50,000 \\
Bank of China & $\mathrm{Li}, \mathrm{He}$ & 3500 \\
$\ldots$ & $\ldots$ & $\ldots$ \\
\hline
\end{tabular}

the shortest path between two nodes on. The average path length $L$ is the mean of path length the network between any pair of nodes, which is calculated as follows:

$$
L=\frac{1}{N(N-1)} \sum_{i \neq j} l_{i j}
$$

The average length determines the size of the effective cable network, which reflects the average degree of separation of the network, describes the randomness and dynamics of the network, indicating that the new information and the fastest dissemination speed of relationship between two customers [13]. The longer the shortest path between two customers is, the slower relationship transmission speed will be and the lower the rate of relationship acquisition will be accordingly, but for shorter-distance networks, the network connection will be relatively stronger with a stronger stability.

\subsection{Clustering Coefficient}

Clustering coefficient describes the network connection nodes in the network between neighboring nodes connected to measure the likelihood of any one of the network nodes. In other words, it is the ratio that neighboring nodes are actually connected to edges and the theoretical side edges [14] [15]. Briefly, the friend of a friend is the probability of your friend. Clustering coefficient $C_{i}$ of node $i$ describes the connection between the nodes in 
the network directly connected to the node, which can be defined as: To account for all possible number of edges in the ratio of the number of edges between nodes directly adjacent to the existing $I$, for edges $=k_{i}\left(k_{i}-1\right) / 2$, we get:

$$
C_{i}=\frac{2 E_{i}}{k_{i}\left(k_{i}-1\right)}
$$

Thus, clustering coefficient of the entire network refers to the arithmetic mean of all nodes' clustering coefficients, we get:

$$
C=\frac{1}{N} \sum_{N}^{1} C_{i}
$$

In the above formula, $N$ is the number of nodes in the network. The average clustering coefficient is an important global indicator in a complex network. Obviously, $C \in[0,1]$. When $C=0$, all nodes are isolated points. When $C=1$, the network between any two nodes is directly connected.

\subsection{Degree Distribution}

The node of degree distribution is an important statistical property for network. A node is the number of other nodes directly connected to the node. We define the degree of node degree distribution $p(k)$ as the probability of node $k$. It reflects the complexity of the network connection between nodes and edges, and it is the overall description of the nature of the network.

\subsection{Results and Analysis}

Obtained by calculation:

The number of nodes of the network is 508, the average path length is 4.3 , and the clustering coefficient is 0.05. Figure 2 shows that the formula of degree distribution for the $p(k)=0.27 k^{-0.88}$ is a power law distribution.

This network has 508 nodes, and the average path length is 4.3, which shows that the customers' relationship is close. The clustering coefficient is only 0.05, indicating that the direct relationship shows the evacuation among the authors. Viewed from above data we find that the relationship between these network customers is primarily indirect. It means only few customers maintain the overall relationship of the network, that is to say, they may have greater influence in the network. By observing the degree distribution and based on the characteristics of power law distribution we finally get this conclusion.

\section{Centrality Analysis of Social Network Model}

Centrality is one of the main analysis indicators of social network analysis methods. Researchers often use degree centrality $\left(C_{D}\right)$ to check the actors' position in the network, which, to some extent, tells whether the actors are important or not and measures their superiority and social status and reputation [16] [17].

Degree centrality, by calculating the point of the degree, makes clear who is the central character in the team, that is, who is the most powerful and influential person in the team. If a point is directly connected with a number of other points, it will have a higher $C_{D}$. In other words, this point will be in the center of the network, being closer to the other actors in the network. We use the number of contact to indicate the degree of the nodes, for node $=a_{i}$, the degree of the nodes $=d\left(a_{i}\right)$, we get:

$$
C_{D}\left(a_{i}\right)=\frac{d\left(a_{i}\right)}{N-1}
$$

In the above formula: $N=$ total network journals (node); $N-1=$ the maximum degree of any node.

While measuring the degree centrality on the basis of the node degree, we should note that this measurement does not take the information known to the node itself into consideration, which may affect the validity of the conclusions. Therefore, we improve the degree centrality, adding the concept of node weights to it.

In practice, the influence of a person (nodes) in social networks depends not only on the number of his or her friends in the network (degrees), also his or her personal capacity (weights) largely. In addition, it may be affected by his or her capacity of direct contact with others, or even may be affected by those are in indirect con- 


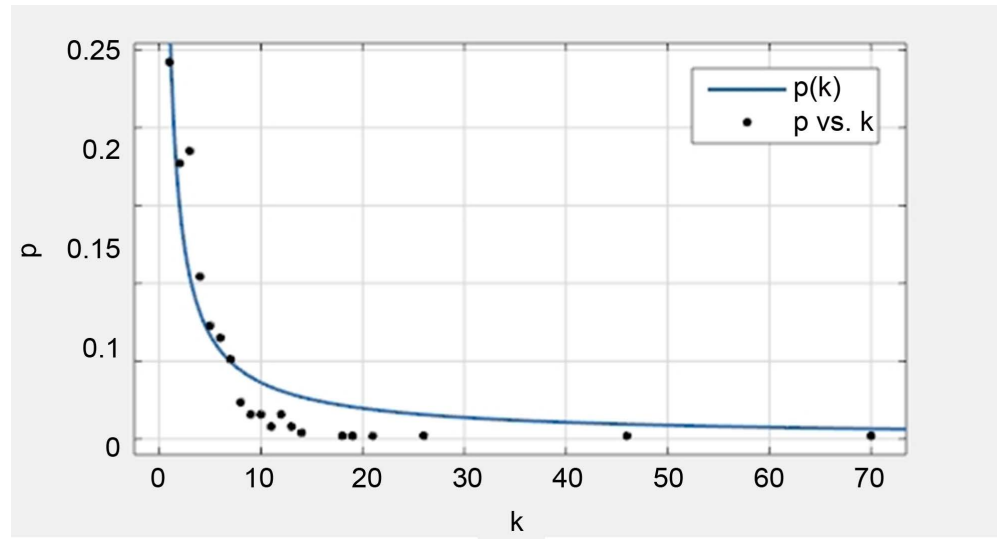

Figure 2. Degree distribution.

tact with him or her.

So, $w_{i}$ is made to serve as the weights of the node $a_{i}$ considering the influence by the weights of the direct link node $a_{i}$ of upon $a_{i}$. Modifying the Formula (4), we get:

$$
C_{w}\left(a_{i}\right)=w_{i} \frac{\sum_{j=1}^{m} w_{i j}}{N-1}
$$

In the Formula (5):

$C_{w}\left(a_{i}\right)$ is the weights that have a direct link between $a_{i}$ and the node set.

$w_{i j}$ and $a_{i}$ are directly connected with the right node weight $a_{i j}$.

$m$ is degree of $a_{i}$.

\section{Conclusions}

Social network analysis has been an important part of social studies. Social network analysis methods employed by this paper to conduct customers' network analysis from the average path length, the clustering coefficient, the centrality and the calculation method are hereby improved. Meantime, this paper puts forward weighting centrality, providing a brand-new perspective for customers' network analysis.

As the data used this paper are accurate and easy to obtain, it guarantees the accuracy of the model to some extent. Our model effectively achieves all of the goals we have set initially. It is fast and can handle large quantities of data. The main advantage of our model is that it has great flexibility, and its calculation method is simple, easy to be spread to other areas and fields.

While our approaches and models can extend and expand the results, there remain several types of weaknesses in our models:

Some special data can't be found, so we have to do some proper assumptions before the solution is found for our models. A more abundant data resource can guarantee a better result in our models.

\section{Acknowledgements}

This work was supported by Soft Science Research Project of Zhejiang Province (2014C35060), Department of Education of Zhejiang Province's Research Project (Y201430369), Zhejiang Provincial Natural Science Foundation of China (LQ13D010001).

\section{References}

[1] Luo, Y.H. (2009) Research and Application of Scale-Free Network Model. Master Thesis, Donghua University, Shanghai.

[2] Ding, S.M. (2012) Social Network Analysis Methods Coauthored Network Applications for Example. Master Thesis, Tianjin Normal University, Tianjin. 
[3] Tao, N.H. and Zhao, X. (2009) The Center of The Indicators and Improvement in the Use of Journal Citation Network Analysis. Library and Information Work, 53, 7-144.

[4] Li, L. (2010) Co-Authored Research Papers and Research Cooperation between Network Structures. Master Thesis, Jilin University, Jilin.

[5] Du, H.F., Li, Z., et al. (2007) Community Structure in Small-World Networks and Scale-Free Networks. Physics, 11, 431-434.

[6] Newman, M.E. (2001) The Structure of Scientific Collaboration Networks. Proceedings of the National Academy of Sciences, 98, 404-409. http://dx.doi.org/10.1073/pnas.98.2.404

[7] Newman, M.E. (2001) Scientific Collaboration Networks. I. Network Construction and Fundamental Results. Physical review E, 64, 8 p. http://dx.doi.org/10.1103/PhysRevE.64.016131

[8] Geng, Z.J. and Wang, W.D. (2009) Analysis of the Cause of the Power-Law of Citation Network. Journal of Intelligence, 28, 15-17.

[9] Zuo, M.Y., Wen, X.W. and Hua, X.Q. (2012) Analysis on Co-Authorship Network of Scholars in Knowledge Management. Journal of Information Resources Management, 4, 4-15.

[10] Erdős, P. and Rényi, A. (1960) On the Evolution of Random Graphs. Publication of the Mathematical Institute of the Hungarian Academy of Sciences, 5, 17-61.

[11] Albert, R., Jeong, H. and Barabási, A.L. (1999) Internet: Diameter of the World-Wide Web. Nature, 401, $130-131$. http://dx.doi.org/10.1038/43601

[12] Barabási, A.L. and Albert, R. (1999) Emergence of Scaling in Random Networks. Science, 286, 509-512. http://dx.doi.org/10.1126/science.286.5439.509

[13] Otte, E. and Rousseau, R. (2002) Social Network Analysis: A Powerful Strategy, Also for the Information Sciences. Journal of Information Science, 28, 441-453. http://dx.doi.org/10.1177/016555150202800601

[14] Bar-Ilan, J. (2008) Informetrics at the Beginning of the 21st Century-A Review. Journal of Informetrics, 2, 1-52. http://dx.doi.org/10.1016/j.joi.2007.11.001

[15] Everett, M.G., Sinclair, P. and Dankelmann, P. (2004) Some Centrality Results New and Old. Journal of Mathematical Sociology, 28, 215-227. http://dx.doi.org/10.1080/00222500490516671

[16] Carrington, P.J., Scott, J. and Wasserman, S. (2005) Models and Methods in Social Network Analysis. No. 28, Cambridge University Press, Cambridge. http://dx.doi.org/10.1017/CBO9780511811395

[17] Freeman, L.C. (1979) Centrality in Social Networks Conceptual Clarification. Social Networks, 1, 215-239. http://dx.doi.org/10.1016/0378-8733(78)90021-7 